\title{
Universal mouse reference RNA derived from neonatal mice
}

\author{
Xiao-Rui He, Chunlian Zhang, and Cam Patterson \\ University of North Carolina, Chapel Hill, NC, USA
}

BioTechniques 37:464-468 (September 2004)

A reproducible, transcriptionally diverse common reference $R N A$ is required for accurate comparisons of data generated from most spotted microarray experiments in different experiments. Several methods have been proposed to make such a reference RNA, such as pooling RNAs isolated from multiple cell lines or tissues, amplifying pooled RNAs, or synthesizing RNAs or DNAs complementary to microarray features. We report an approach to prepare a large amount of mouse reference RNA from whole neonatal mice. This approach is simple, quick, reliable, reproducible, and inexpensive. The whole mouse reference RNA is highly representative when compared to two commercially available universal mouse reference RNAs isolated and pooled from multiple cell lines or organs.

\section{INTRODUCTION}

Microarray techniques have been widely used to monitor large-scale gene expression simultaneously in multiple specimens. After a proper integration of gene expression data, we may not only be able to evaluate the differential expression of genes under various conditions, but also be able to identify coordinated gene expression profiles (1-3). One critical technical issue that impinges on the integration of microarray data is that the measured intensity of a labeled probe [synthesized cDNA using messenger RNA (mRNA) in samples as template] reflects not only the biological variation, but also bias induced by factors such as the sequence, density, size, and shape of the corresponding target [synthesized oligonucleotide designed from the sequence of a known gene transcript or expressed sequence tag (EST)] loaded on the microarrays. Other factors, including probe labeling and hybridization, may also bias the measurement of the spot intensity. These interfering factors can be largely corrected by the two-channel method, which usually includes separately labeled sample and reference RNAs (3-6). Since the probes prepared from the sample and the reference are hybridized and measured in the same experiment, the ratio of sample signal to reference signal may diminish the biases and thus intensify the biological variation. A disadvantage of representing data by using sampleto-reference ratio is that the value of the ratio can be exaggerated when a denominator is too small. Therefore, spot representation or coverage, which indicates the number of mRNAs whose concentration is above a reasonable level in a sample, should be considered when choosing a reference mRNA for two-channel microarray experiments.

Tissues or cells have characteristic gene expression patterns, and only a subset of all genes may be expressed at a level that can be used as a reference for microarray experiments. Therefore, pooling RNA isolated from different tissues or cell lines together is a reasonable strategy to obtain a reference RNA with broader representation (710). A disadvantage of this strategy is that a reference RNA prepared in one laboratory is difficult to replicate in other laboratories or even by the same laboratory at different times because of restrictions introduced by materials and conditions. We introduce a simple procedure to prepare a large amount of highly representative, reproducible, and inexpensive reference RNA from whole neonatal mice.

\section{MATERIALS AND METHODS}

\section{Sample Collection}

One-day-old mice of two strains (129SvEv and C57BL/6; Charles River Lab, Wilmington, MA, USA) were collected. Animals were sacrificed by isoflurane inhalation, frozen in liquid nitrogen, and stored at $-80^{\circ} \mathrm{C}$.

\section{RNA Isolation}

Frozen mice were placed into 10 $\mathrm{mL}$ of TRIzOL ${ }^{\circledR}$ reagent (Invitrogen, Carlsbad, CA, USA) per gram of tissue in 50-mL screw cap tubes and immediately homogenized with Polytron PT10 (Mbcoct, New Haven, CT, USA) at the highest speed at room temperature for $1 \mathrm{~min}$. The homogenized sample was incubated at room temperature for $5 \mathrm{~min}$ and then centrifuged at $500 \times \mathrm{g}$ at $4^{\circ} \mathrm{C}$ for $5 \mathrm{~min}$. The upper fat layer was removed, and sample was transferred to $15-\mathrm{mL}$ DNase- and RNasefree screw cap tubes. After adding 0.2 $\mathrm{mL}$ chloroform per $1 \mathrm{~mL}$ TRIzoL, the tube was shaken vigorously for $30 \mathrm{~s}$ and incubated at room temperature for $5 \mathrm{~min}$. The mixture was centrifuged at $4^{\circ} \mathrm{C}$ at $10,000 \times g$ for $18 \mathrm{~min}$, the aqueous phase was transferred to a new 15$\mathrm{mL}$ tube, and RNA was precipitated by adding $0.5 \mathrm{~mL}$ isopropyl alcohol per 1 $\mathrm{mL}$ TRIzOL. The mixture was incubated at room temperature for $10 \mathrm{~min}$ and then centrifuged at $10,000 \times g$ at $4^{\circ} \mathrm{C}$ for $10 \mathrm{~min}$. The supernatant was removed, and the RNA pellet was washed with $75 \%$ ethanol. The pellet was air-dried for 10-20 min at room temperature and then resuspended in $1 \mathrm{~mL}$ DNase- and RNase-free water and stored at $-80^{\circ} \mathrm{C}$. The quantity of total RNA was determined by measuring $\mathrm{A}_{260}$, and the quality of the total RNA was determined with the RNA Nano LabChip ${ }^{\circledR}$ and Agilent 2100 Bioanalyzer (Agilent Technologies, Wilmington, DE, USA).

\section{Microarrays, Probe Labeling, Hybridization, and Data Analysis}

Two commercially available universal mouse reference RNAs (UMRR) produced by Stratagene (UMRR-S; La Jolla, CA, USA) and BD Biosciences (UMRR-BD; Palo Alto, CA, USA) were used as comparisons with RNA isolated from whole neonatal mice. For each sample, $40 \mu \mathrm{g}$ total RNA was used for preparing cDNA probes labeled with Cy ${ }^{\mathrm{TM}} 3$ or Cy5. Mixtures of the two fluorescence dye-labeled probes originating from the same reference RNA or from different reference RNAs were hybrid- 
ized to 22K Mouse Development Oligo Microarrays (Agilent Technologies). Microarrays were scanned using the GenePix 4000B scanner (Axon Instruments, Union City, CA, USA) with photomultiplier tube (PMT) setting at 540. Images were quantified with GenePix Pro 4.1 software (Axon Instruments).

\section{RESULTS AND DISCUSSION}

\section{Quality and Quantity of Total RNA Harvested from Neonatal Mice}

The quality of total RNA isolated from individual mice was determined using the RNA Nano LabChip. The electropherogram of pooled total RNA is shown in Figure 1A. RNAs originated from four groups of mice with equal numbers $(n=5)$ of male 129SvEv mice, female 129SvEv mice, male C57BL/6 mice, and female C57BL/6 mice. In each group, mice were collected from two to four litters. The number of mice originated from the same litter was one to three. The mice in the same litter were housed in the same cage, and the mice in different litters were housed in different cages and had different dams. Two batches of pooled RNAs were prepared separately by using the same approach at different times. The total RNA yield was proportional to the body weight (Figure 1B). The average body weight \pm SD of mice was $1.43 \pm$ $0.18 \mathrm{~g}$, and the average total RNA $\pm \mathrm{SD}$ yield from each mouse was $4.98 \pm 0.66$ mg. An equal amount of total RNA harvested from each mouse was added together, so that each mouse had the same contribution to the pooled reference RNA. We harvested 78 and $80 \mathrm{mg}$ of pooled total RNA from two batches of mice, which are sufficient for 3950 microarray experiments using $40 \mu \mathrm{g}$ total RNA per experiment.

\section{A}

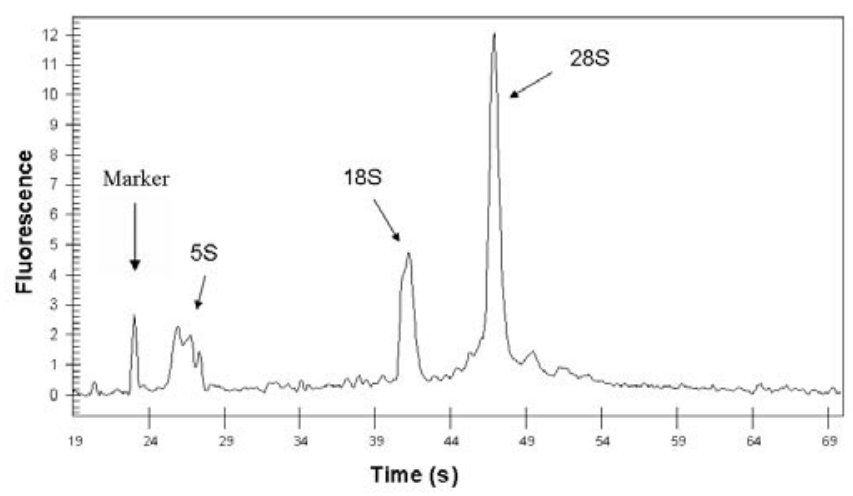

B

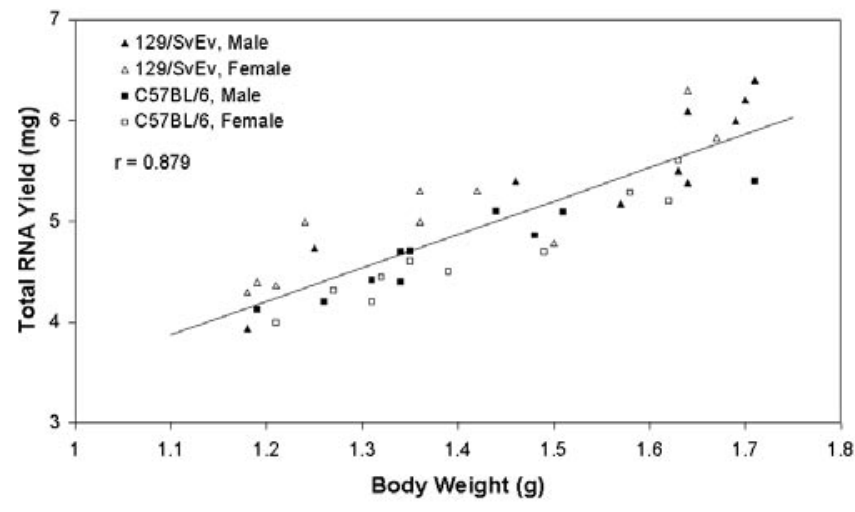

Figure 1. Quality and quantity of total RNA harvested from neonatal mice. (A) An electropherogram of pooled total RNA. Three ribosomal peaks, 5S, 18S, and 28S, are indicated. (B) Relationship between the amount of total RNA isolated and mouse body weight. Total RNA was extracted with TRIzoL reagent from four groups ( $n=10$ for each) of mice of indicated strains and sexes. 


\section{Comparison of the Gene Representation Between Whole Mouse Reference RNA and UMRR-BD or UMRR-S}

A mixture of Cy3-labeled whole mouse reference RNA (WMRR) and Cy5-labeled UMRR-BD or UMRR-S was hybridized to a set of microarrays. Conversely, a mixture of Cy5-labeled WMRR and Cy3-labeled UMRR-BD or UMRR-S was hybridized to another set of microarrays. All microarrays were scanned with a fixed PMT under which spots were not saturated. The minimal spot intensity was set at 1 , and the ratio of mean intensity of two channels was normalized to 1 . The average intensity of each spot for a particular reference RNA was calculated from both $\mathrm{Cy} 3$ and $\mathrm{Cy} 5$ channels in a pair of corresponding microarrays. The distributions of low spots for WMRR versus UMRR-BD and for WMRR versus UMRR-S are shown in Figure 2, A and B. The number of spots for which the average intensity was lower than 100 was 93 fewer in WMRR compared to
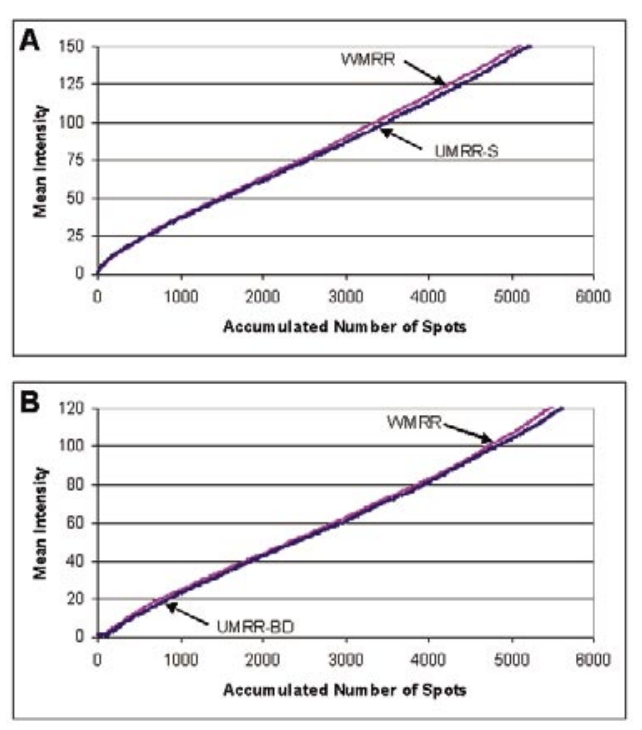

Figure 2. Distribution of spots with low intensity in Agilent 22K Mouse Development Oligo Microarrays. Three RNA samples were used to prepare cDNA probes labeled with both $\mathrm{Cy} 3$ and $\mathrm{Cy} 5$ dyes. Data were the average calculated from the paired dye-swap microarrays. (A) Different labeled probes of WMRR and UMRR-S were hybridized to the same microarrays. (B) Different labeled probes of WMRR and UMRR-BD were hybridized to the same microarrays. WMRR, the RNAs isolated from whole neonatal mice; UMRR-BD, the universal mouse reference RNA from BD Biosciences; UMRR-S, the universal mouse reference RNA from Stratagene.
UMRR-BD in a pair of dye-swap microarrays and was 131 fewer in WMRR compared to UMRR-S in another pair of dye swap microarrays. However, these differences were not statistically significant by $\chi^{2}$ test.

\section{Reproducibility of WMRR}

The evaluation of the reproducibility of WMRR in different batches was achieved by measuring the biological contribution to variability in our microarray experiments. In this group of experiments, data were normalized using LOWESS with software MIDAS2.17 (11). Two batches of WMRR (WMRR-a and WMRR-b) were used to prepare cDNA probes labeled with Cy3 and Cy5 separately. Three mixtures of different combinations of probes were hybridized to three microarrays. The combinations were: (i) Cy3-labeled probes from WMRR-a versus Cy5-labeled probes from WMRR-a; (ii) Cy3labeled probes from WMRR-b versus Cy5-labeled probes from WMRR-b; and (iii) Cy3-labeled probes from WMRR-b versus Cy5-labeled probes from WMRR-a. The results are shown in Figure 3 , A-C. The first and second combinations reveal the variation from nonbiological sources. The variation is inversely related to the tightness of the linear relationship between two channels. The third combination reveals the variation from both biological and nonbiological sources. A high similarity of data distributions and correlation coefficients between the third combination and the two other combinations indicates that the difference between two batches of WMRR is negligible.

The reference RNAs from difference sources were also compared. Two mixtures of Cy3-labeled probes from WMRR-a versus Cy5-labeled probes from UMRR-BD and Cy3-labeled probes from WMRR-a versus Cy5-labeled probes from UMRR-S were hybridized to two microarrays, respectively (Figure 3, C and D). In contrast to the comparison between WMRR- $a$ and WMRR- $b$, there are greater differences between WMRR and UMRR-BD or UMRR-S.

The ideal reference RNA should be highly representative, evenly distributed, highly reproducible, inexpensive, and easy to prepare. No method has yet been found to make such an ideal reference RNA. Synthesized oligonucleotides can be evenly mixed and in theory should be able to cover all genes represented on a microarray (12). Similarly, a common reference consisting of a mixture of the cDNA PCR products that are spotted on particular microarrays has high coverage for the same microarrays (13). However, the specific design of oligonucleotides or cDNAs narrows the range of microarray platforms to which they can be applied. Although endogenous mRNAs isolated from a particular tissue or cell line will have narrow coverage and uneven distribution, pooling mRNAs isolated from multiple tissues and/or cell lines helps to reduce these disadvantages by a certain amount. Using pooled RNA as a reference, it is not only possible to compensate for variances that result from the uneven loading of targets on microarrays, but it is also possible to compensate for variances that result from other sources such as amplification, labeling, and hybridization.

The idea of "reference" RNA was initiated in pioneering microarray experiments that used one RNA sample in a series of seven samples in a yeast metabolic study so that every experiment was compared to a common RNA sample (14). This idea has been widely used and modified, and many models of reference design have been introduced (15-17). For example, each sample is tested against other samples in sequence, in loop, or in a mixed sequential/loop format. This use of relative references and complex design of experiments can increase the efficiency and decrease the variation in a well-planned study. However, the data from multiple studies are difficult to integrate without a common reference. Another commonly encountered problem is that a reference RNA prepared in one laboratory may be difficult, if not impossible, to replicate in other laboratories (13). Thus, dissimilar reference 
RNAs are used in different laboratories for related experiments, which creates difficulty for data integration and for maximizing the value of the microarray data in research communities.

Now, several commercial universal reference RNAs have become available. The large-scale production of
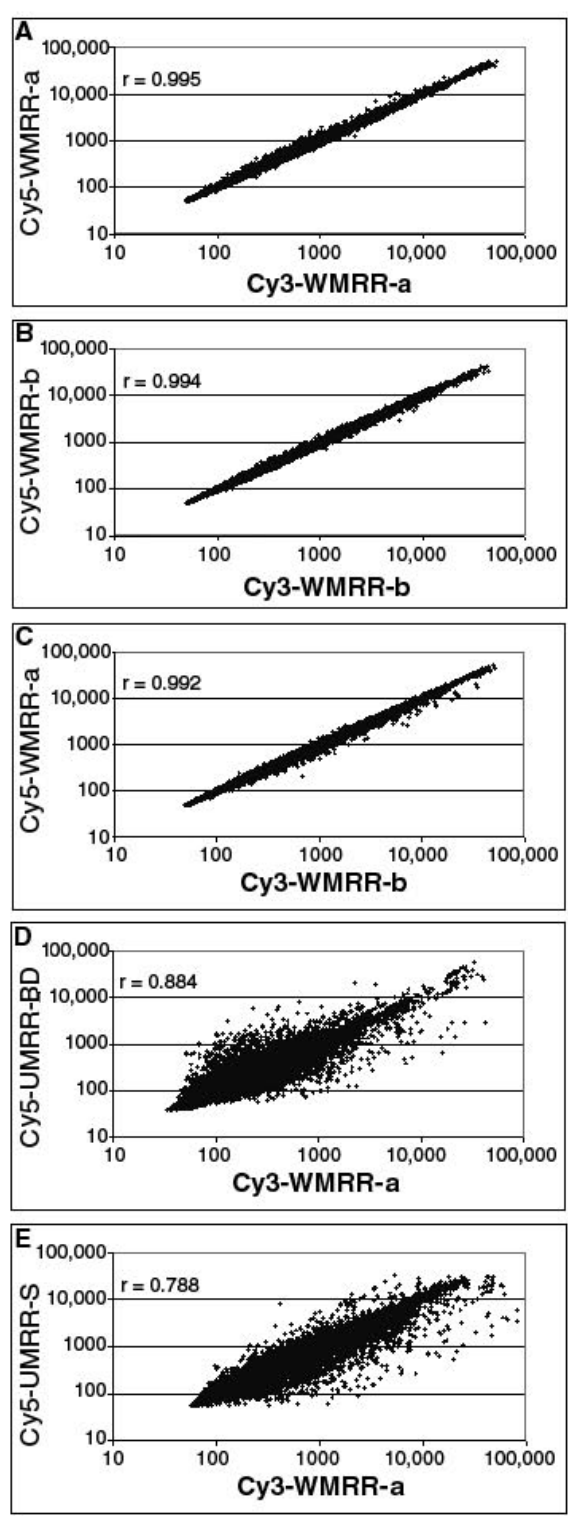

Figure 3. Reproducibility of reference RNAs. (A and B) The consistency of two separately prepared probes labeled with Cy3 and Cy5 from two aliquots of a WMRR (batch a or batch b). (C-E) The similarity between probes prepared from WMRR (batch a) and probes prepared from WMRR (batch b), UMRR-BD, and UMRR-S, respectively. WMRR, the RNAs isolated from whole neonatal mice; UMRR-BD, the universal mouse reference RNA from BD Biosciences; UMRR-S, the universal mouse reference RNA from Stratagene. reference RNA offers a solution for the requirement of unique reference RNA. UMRR-BD and UMRR-S are two samples that use pooling strategies to achieve their high representations and can be used as "universal" references. However, these universal reference RNAs are extremely expensive for routine use, and their complex compositions by pooling the RNAs harvested from multiple cell lines or tissues prevent the preparation of similar reference RNAs by individual laboratories. A less expensive method for obtaining milligrams of reference RNA from micrograms of pooled total RNA by using a double amplification method has been reported previously (18). An advantage of this method is that the amplified RNA products can be further labeled like native RNA. However, this procedure uses a complicated mixture of initial RNAs derived from different cell lines, tissues, and organisms and double amplifications, which increases the fragility of the reproducibility of the reference RNA. The limitations of these approaches for referencing microarray experiments led us to search for alternative approaches.

We have found an effective method to make a common mouse reference RNA from whole neonatal mice that avoids the need for amplification or other bias-introducing procedures. The coverage of this reference RNA is as wide as two UMRRs produced by Stratagene and BD Biosciences. It is not surprising that the RNAs isolated from a whole animal are highly representative, because a body of a whole animal contains different types of cells and tissues. Since the combination of varied cells and tissues as well as their gene expressions in a body is dictated by nature, it is easy to reproduce the RNAs from the same type of age- and sex-matched ani- 
mals. To reduce the individual variances and increase the yield of RNA, we pooled the RNAs isolated from 20 animals and used them as reference RNA. Two strains of widely used mice with both sexes were included to maximize gene representation. Since the yield of RNA from mice was proportional to their body weights, and the body weights are variable, the addition of equal amounts of RNA isolated from individual mice to the pool may facilitate the reproducibility of WMRR. It is also probably judicious to use mice of similar body weight. In our experiment, weights in the range of $1.07-1.79 \mathrm{~g}$ are within 2 sDs of the mean weight.

It is well known that gene expression profiles are different at different developmental stages. To prepare an overall gene representative reference RNA, pooling tissues of animals at different developmental stages is one choice as used in UMRR-S. A feature of the neonatal mouse is that it is at a turning point of its life between an embryo and a mature animal, and its gene expression profiles at this stage may have a wider overlap with both embryonic and adult mice. We have successfully used the whole mouse reference RNA to study gene expression profiles in mouse embryonic stem cells, various mouse cell lines, and adult mouse organs in microarray experiments (data not shown). Compared to the double amplification method reported previously (18), a large amount of WMRR (about $4 \mathrm{mg}$ / mouse) can be made quickly (within 1 day) at a low cost, because the material of neonatal mice and batch size is unrestrictive, and only a simple RNA isolation process is needed without further amplifications. On the other hand, when the material source for RNA is limited, the value of the double amplification method is obvious.

In summary, the preparation of a large amount of mouse reference RNA from whole neonatal mice is simple, quick, reliable, and inexpensive. The mouse reference RNA is highly representative compared to two commercial universal mouse reference RNAs. The native combination of cells and tissues as well as their gene expression in a body favors the replication of this reference RNA.

\section{ACKNOWLEDGMENTS}

This work was supported by National Institutes of Health (NIH) grant no. HL072347 to C.P.

\section{COMPETING INTERESTS STATEMENT}

The authors declare no conflicts of interest.

\section{REFERENCES}

1.Claverie, J.M. 1999. Computational methods for the identification of differential and coordinated gene expression. Hum. Mol. Genet. 8:1821-1832.

2.Karsten, S.L., L.C. Kudo, R. Jackson, C. Sabatti, H.I. Kornblum, and D.H. Geschwind. 2003. Global analysis of gene expression in neural progenitors reveals specific cell-cycle, signaling, and metabolic networks. Dev. Biol. 261:165-182.

3.Perou, C.M., S.S. Jeffrey, M. van de Rijn, C.A. Rees, M.B. Eisen, D.T. Ross, A. Pergamenschikov, C.F. Williams, et al. 1999. Distinctive gene expression patterns in human mammary epithelial cells and breast cancers. Proc. Natl. Acad. Sci. USA 96:9212-9217.

4.Quackenbush, J. 2002. Microarray data normalization and transformation. Nat. Genet. 32(Suppl):496-501.

5.Yang, I.V., E. Chen, J.P. Hasseman, W. Liang, B.C. Frank, S. Wang, V. Sharov, A.I. Saeed, et al. 2002. Within the fold: assessing differential expression measures and reproducibility in microarray assays. Genome Biol. 3:research0062.

6.Yang, Y.H., S. Dudoit, P. Luu, D.M. Lin, V. Peng, J. Ngai, and T.P. Speed. 2002. Normalization for cDNA microarray data: a robust composite method addressing single and multiple slide systematic variation. Nucleic Acids Res. 30:e15.

7.Alizadeh, A.A., M.B. Eisen, R.E. Davis, C. Ma, I.S. Lossos, A. Rosenwald, J.C. Boldrick, H. Sabet, et al. 2000. Distinct types of diffuse large B-cell lymphoma identified by gene expression profiling. Nature 403:503511.

8.Perou, C.M., T. Sorlie, M.B. Eisen, M. van de Rijn, S.S. Jeffrey, C.A. Rees, J.R. Pollack, D.T. Ross, et al. 2000. Molecular portraits of human breast tumours. Nature 406:747-752.

9.Scherf, U., D.T. Ross, M. Waltham, L.H. Smith, J.K. Lee, L. Tanabe, K.W. Kohn, W.C. Reinhold, et al. 2000. A gene expression database for the molecular pharmacology of cancer. Nat. Genet. 24:236-244.

10.van 't Veer, L.J., H. Dai, M.J. van de Vijver, Y.D. He, A.A. Hart, M. Mao, H.L. Peterse, K. van der Kooy, et al. 2002. Gene expression profiling predicts clinical outcome of breast cancer. Nature 415:530-536.

11.Saeed, A.I., V. Sharov, J. White, J. Li, W.
Liang, N. Bhagabati, J. Braisted, M. Klapa, et al. 2003. TM4: a free, open-source system for microarray data management and analysis. BioTechniques 34:374-378.

12.Dudley, A.M., J. Aach, M.A. Steffen, and G.M. Church. 2002. Measuring absolute expression with microarrays with a calibrated reference sample and an extended signal intensity range. Proc. Natl. Acad. Sci. USA 99:7554-7559.

13.Sterrenburg, E., R. Turk, J.M. Boer, G.B. van Ommen, and J.T. den Dunnen. 2002. A common reference for cDNA microarray hybridizations. Nucleic Acids Res. 30:e116.

14.DeRisi, J.L., V.R. Iyer, and P.O. Brown 1997. Exploring the metabolic and genetic control of gene expression on a genomic scale. Science 278:680-686.

15.Kerr, M.K. and G.A. Churchill. 2001. Statistical design and the analysis of gene expression microarray data. Genet. Res. 77:123128.

16.Townsend, J.P. 2003. Multifactorial experimental design and the transitivity of ratios with spotted DNA microarrays. BMC Genomics 4:41

17.Yang, Y.H. and T. Speed. 2002. Design issues for cDNA microarray experiments. Nat. Rev. Genet. 3:579-588.

18.Puskas, L.G., A. Zvara, L. Hackler, Jr., T. Micsik, and P. van Hummelen. 2002. Production of bulk amounts of universal RNA for DNA microarrays. BioTechniques 33:898904.

Received 11 December 2003; accepted 13 May 2004.

Address correspondence to Cam Patterson, Carolina Cardiovascular Biology Center, University of North Carolina at Chapel Hill, 8200 Medical Biomolecular Research Building, Chapel Hill, NC 27599-7126, USA.e-mail: cpatters@med.unc.edu 